\title{
The Processing Algorithm for Wideband Signals Propagating in Media with Frequency Dispersion
}

\author{
Andrei A. Kalshchikov, Student Member IEEE, Vitaly V. Shtykov and Sergey M. Smolskiy, Member IEEE ${ }^{l}$
}

\begin{abstract}
The new processing algorithm for wideband signals, which are propagating in frequency dispersion media, is developed in the context of the ground penetrating radar technology. The mathematical substantiation of offered method is presented on the base of linear functional spaces. The method is described for calculation acceleration on the base of the recursive approach. Results of numerical modeling of this method are presented for electromagnetic waves propagating in frequency dispersion media at utilization of chirp pulse signals. The Debye model is used as the model of electrical medium properties. The frequency dispersion of losses in the medium is taken into consideration at modeling. Results of operation modeling of the ground penetrating radar are described. Results of this paper will be useful for the ground penetrating radar technology and the ultrasonic flaw detection.
\end{abstract}

Index Terms-phase velocity, frequency dispersion, phase correction, ground penetrating radar technology, ultrasonic flaw inspection.

\section{INTRODUCTION}

An increase of the radio systems noise-immunity and effectiveness takes the central place in the modern theory and engineering of information transmission. During the growth of the information transmission rate, we see the effect increase of dispersion characteristics of the communication channel. This increase is manifested in distortions of the transmitting signal shape. These distortions can make impossible to extract of transmitted information from the received signal.

The complex transfer function of the channel route with the length $L[1]$ for the plain wave is

$$
K(j \omega, L)=\exp [-j \gamma(\omega) L],
$$

where $\gamma(\omega)=\beta(\omega)-j \alpha(\omega)$ is the complex propagation constant, $\beta(\omega)$ is the phase constant, $\alpha(\omega)$ is the damping constant.

Signal distortions after passage through the channel route are caused by disturbances of the amplitude-phase relations between its spectral components.

Andrei A. Kalshchikov is with Moscow Power Engineering Institute (National Research University), Russia (e-mail: KalschikovAA@gmail.com).

Vitaliy V. Shtykov is with Moscow Power Engineering Institute (National Research University), Russia (e-mail: ShtykovVV@yandex.ru)

Sergey M. Smolskiy is with Moscow Power Engineering Institute (National Research University), Russia (e-mail: SmolskiySM@mail.ru)
The phase constant $\beta(\omega)$ in (1) can be expanded into the power series around the frequency of the carrier oscillation $\omega_{0}$ :

$$
\beta(\omega)=\beta\left(\omega_{0}\right)+\frac{d \beta}{d \omega} \Omega+\frac{d^{2} \beta}{2 d \omega^{2}} \Omega^{2}+\ldots \operatorname{Rem}_{n}(\Omega) .
$$

where $\Omega=\omega-\omega_{0}, \operatorname{Rem}_{n}(\Omega) \leq \frac{|\Omega|^{n}}{n !} \sup _{0<\xi<\Omega}\left|\frac{d^{n} h}{d \omega^{n}}\right|_{\xi} \mid$ is the series remainder.

For the case of the weak dispersion, we may be limited by the first term and can introduce the group velocity:

$$
V_{g r}(\omega)=\frac{d \omega}{d \beta} .
$$

For the channel route of the given length $L$, we can introduce the group delay time:

$$
\tau_{g r}=\frac{d \beta}{d \omega} L
$$

With this very convenient approximation, the signal envelope (at bottom of fact, information) propagates through the channel route with the group velocity practically without distortions.

Using $\operatorname{Rem}_{n}(\Omega)$, we can estimate the relative error of this approximation as follows:

$$
\left.\delta_{\beta} \approx \delta_{\Omega} \omega_{0}\left|\frac{d^{2} \beta}{d \omega^{2}}\right|_{\omega_{0}}|/| \frac{d \beta}{d \omega}\right|_{\omega_{0}} \mid,
$$

where $\delta_{\Omega}$ is the relative frequency bandwidth of the signal spectrum.

However, this condition is necessary but not sufficient. The thing is that the real and imaginary parts of the substance permittivity are connected with each other by the KramersKronig relations. This means that the dispersion of the phase velocity inevitably accompanies by the damping index $\alpha(\omega)$ dispersion. Therefore, for the relevancy of the group velocity approximation, the following equality is necessary:

$$
\delta_{\alpha}=\delta_{\Omega} \frac{\omega_{0}}{\alpha\left(\omega_{0}\right)} \frac{d \alpha}{d \omega} .
$$

With transfer to wideband and ultra-wideband signals, the error of the group velocity approximation becomes so much that its application becomes impossible at signal processing.

In traditional radar technology with strongly expressed frequency dispersion, we can meet not very often, for example, in tasks of electromagnetic wave propagation in an ionosphere [2], or in tasks of the radar ground penetrating sensing $[3,4]$. In the sonar technology and especially in the 
ultrasonic flaw inspection, we can practically often meet with the frequency dispersion of the elastic wave velocity.

Firstly, the frequency dispersion leads to the shape distortion of the probing pulse, and secondly, leads to disturbance of the direct proportional connection between the delay time of the reflected signal and the distance to the sensing object [5]. In the radar ground penetrating technology, this complicates the deciphering of received data. The problem of the phase velocity dispersion influence is discussed in $[6,7,8]$. The necessity to develop the signal processing methods, which can decrease its distortions and increase the accuracy of objects' sensing remains very relevant. In this paper, we describe of the one of possible methods of signal processing at the presence of the frequency dispersion in the route of its propagation.

\section{MATHEMATiCAL SUBSTANTIATION OF THE OFFERED METHOD}

If the phase constant $\beta(\omega)$ and the route length $L$ are known, which is typical for the communication channel, the task of signal distortion correction can be solved. To decrease the influence of the frequency dispersion, different methods of the filter-corrector synthesis are actively developed both in time and frequency domains. For the transmission line without losses, such a filter, as a matter of fact, represents the phase corrector with the phase-frequency response of the following type:

$$
\Phi_{\text {cor }}(\omega)=\beta(\omega) L .
$$

The task can be also solved if the transmitted signal shape and $p(\omega)$ are given, while the route length $L$ is unknown and is a subject to determination. In this case, the initial signal reconstruction is not required, and $L$ (the sensing object position) can be determined using the cross-correlation function $\Psi(t, L, z)$ of the received signal $r(t, L)$ and the trial function - the signal model $s(t, z)$ :

$$
\Psi(t, L, z)=r(t, L) * s(t, z) .
$$

This situation is typical for manifold radar systems.

Considering (4) as an equation for reconstruction of the $r(t, L)$ function, we come to the known procedure of the deconvolution.

The function $\Psi(t, L, z)$ represents the surface above the plane $\langle t 0 z\rangle$. The determination of the $L$ value is reduced to the search of the global maximum position of this surface: $\Psi_{M}\left(t_{M}, z_{M}=L\right)$. However, such the search procedure requires the large time expenses and the large volume of the processor memory, while the abscissa value $t_{M}$ does not any interest for us. Therefore, we can try to accelerate this procedure by excluding the variable $t$ from the equation (4).

\section{INITIAL RELATIONSHIPS OF THE OFFERED ALGORITHM}

In order to exclude time from the equation (4), we make use the generalized signal theory [8].
Let us form on the base of trial functions $s(t, z)$ the $\left.M=\left\{s\left(t, z_{1}\right), s\left(t, z_{2}\right), s\left(t, z_{3}\right), \ldots\right)\right\}$ infinite-dimension linear set, which, in the general case, forms the linear, complex functional space $[8,9]$. In this space, we can form the scalar product of the signal and the trial function:

$$
W(z)=\int_{-\infty}^{\infty} r(t, L) s^{*}(t, z) d t,
$$

which, in essence, represents the mutual energy. According to the Cauchy-Schwarz inequality:

$$
|W(z)|^{2} \leq\|r\|^{2} \cdot\|s\|^{2}
$$

where

$$
\|r\|^{2}=\left|\int_{-\infty}^{\infty} r(t, L) r^{*}(t, L) d t\right|
$$

and

$\|s\|^{2}=\left|\int_{-\infty}^{\infty} s(t, z) s^{*}(t, z) d t\right|$ is the energy of the signal and the trial function, relatively.

The equality is fulfilled if:

$$
s(t, L) \equiv r(t, z) \text {. }
$$

Thus, the variation of the functional (5) solves the problem of $L$ determination.

If to make use the generalized Rayleigh's energy theorem [8], then the functional (5) can be transformed to the form:

$$
W(z)=\int_{-\infty}^{\infty} R(\omega, L) S^{*}(\omega, z) d \omega
$$

where $R(\omega, L)$ and $S(\omega, z)$ are spectral densities of the signal and the trial function, relatively.

If $\gamma(\omega)$ is known, and the shape of the transmitted signal - the probing pulse (PP) $y(t)$ and its spectral density $Y(\omega)$ - are known, the spectral density of the trial function is:

$$
S(\omega, z)=Y(\omega) \exp [-j 2 \xi(\omega) z] \text {. }
$$

Substituting this in (6), we obtain:

$$
W(z)=\int_{-\infty}^{\infty} R(\omega, L) \dot{Y}^{*}(\omega) \exp [j 2 \vartheta(\omega) z] d \omega .
$$

Thus, the procedure of the distance search to the object can be performed both in the space-time (6) and the frequency domains (7). In the first case, it is necessary to transform the trial function spectrum $S(\omega, z)$ into the pulse characteristic of the filter-corrector $h(t, z)$. In the second case, we need to transform the received signal $r(t, L)$ into the spectral function $R(\omega, L)$.

\section{THE SOFTWARE REALIZATION OF THE ALGORITHM}

Functionals (6) and (7) do not contain time in the explicit form. Therefore, at digital implementation of the algorithm, the operation of $L$ determination can be performed 
The Processing Algorithm for Wideband Signals

Propagating in Media with Frequency Dispersion independently on the operation of other parts of the system, using stored in the separate memory buffer of the signal, which is received on the next stage of the radar operation.

Since the object position is unknown in advance and the receiver signal shape differs from $\mathrm{PP}$, the search in the distance can be performed only on the signal sample with the duration not less then $T_{D} \leq 2 D / V_{g r}$, where $D$ and $V_{g r}$ are the system operation distance and the group velocity on the central frequency, relatively.

The functional flow block-diagram is shown in Figure 1.

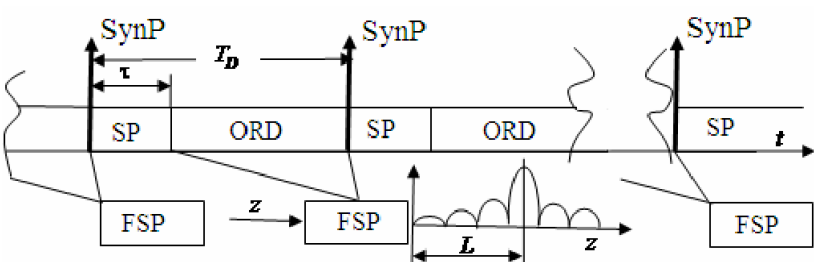

Figure 1. The functional flow block-diagram of the system: SynP is the synchronization pulse, SP is the radiation interval $\tau$ of the sensing pulse, ODR is the operation distance range, FSP is the flow of signal processing

The upper part of the diagram represents the flow of data recording, which are recorded into the memory buffer for the further parallel procesing.

As the example, we examine the spectral method of the object position determination. The functional (6) in the discrete form has the form:

$$
W_{n}=\Delta \omega \sum_{m=0}^{M-1} R_{m} S_{m, n}^{*},
$$

where $\quad R_{m}=R\left(\omega_{m}\right), \quad S_{m, n}=Y\left(\omega_{m}\right) \exp \left(-j 2 \gamma_{m} n \Delta z\right)$, $n=\overline{0, N-1}$. For discrete samples $W_{n}$ the recerrent relation acts:

$$
W_{n+1}=\Delta \omega \sum_{m=0}^{M-1} W_{n} \exp \left(-j 2 \gamma_{m} \Delta z\right),
$$

which allows the accelreration of the functional values calculation.

The number of samples $M$ and the discretization frequency $f_{d}=1 / \tau_{d}$ are determined as usual. As to the the step in $\Delta z$, it is determined by the spatial spread of the SP autocorrelation function.

\section{PRELIMINARY PREREQUISITES TO THE ALGORITHM MODELING}

In the ground penetrating radar (GPR), two operating modes are used $[3,4,10]$ : the pulse mode and the Continuous Wave GPR (CW-GPR) mode. Both in the first and in the second case, the ultra-wideband sensing pulses are used.

In the pulse GPR, these are radio pulses containing the pair or of periods of the carrier frequency. In CW-GPR, the signal with linear frequency modulation is used or the train of short pulses, which frequency varies in the wide limits by steps (the Step Frequency GPR - SF-GPR) [3,10,11].
As the illustration of the offered method, we perform the numerical modeling for the signal with the linear frequency modulation of the following type:

$$
u(t)=U_{m} \exp \left[-(t / \tau)^{2} \cos \left[2 \pi f_{0}(t / \tau)-\pi B(t / \tau)^{2}\right],\right.
$$

where $U_{m}$ is the SP amplitude, $\tau$ is the pulse duration on the level of $e^{-1}, f_{0}$ is the frequency of the carrier oscillation, $B$ the signal base.

The signal choice in the form of (10) is caused by the fact that its' spectral density is expressed through the elementary functions. This allows the presentation of the trial function in the form of the formula avoiding the utilization of the Fourier transform.

For the sake of more generalization achievement, we use further the normalized time $t=t / \tau$, the non-dimension frequency of the carrier oscillation $F_{0}=f_{0} \tau$ and nondimension co-ordinate $z=z / \lambda_{0}$, where $\lambda_{0}=C / f_{0}$ is the wavelength in the vacuum, and $C$ is the light speed.

To describe the electrical properties of the humid soil, we use models, which take into consideration the orientation polarization and the ionic conduction $[3,6,7,12,13]$. In conformity with tasks of the ground penetrating radar, parameters of such models are obtained as the result of the numerous experiments.

The orientation polarization is described by the Debye model:

$$
\varepsilon(\omega)=\varepsilon_{\infty}+\frac{\varepsilon_{s t}-\varepsilon_{\infty}}{1+j \omega \tau_{r l}}=\varepsilon_{\infty}+\frac{\varepsilon_{s t}-\varepsilon_{\infty}}{1+j 2 \pi F \mathrm{~T}_{r l}},
$$

where $\varepsilon_{\infty}$ and $\varepsilon_{s t}$ is the relative permittivity at $\omega \rightarrow \infty$ and $\omega=0$, relatively; $\tau_{r l}$ in the relaxation time of the orientation polarization, $\mathrm{T}_{r l}=\tau_{r l} f_{0}$.

To illustrate the possibilities of the above-described method, we can be limited by the single-component medium model. For numerical modeling, we use parameters of the hypothetic medium presented in [13]. We take in (11): $\varepsilon_{s t}=8.1, \varepsilon_{s t}=5.7, \tau_{r l}=22 \mathrm{ps}$. For the frequency $f_{0}$ about $100 \mathrm{MHz}, \mathrm{T}_{r l}$ has an order of $10^{-3}$.

\section{NUMERICAL MODELING OF THE METHOD}

The modeling software consists of two parts. The first one imitates the SP propagation in the route and operation of the homodyne receiver. The second part is the processing algorithm itself.

At signal modeling $f_{0} \tau=128, B=32\left(\delta_{\Omega}=25 \%\right)$. In the medium model (11) we use $\mathrm{T}_{r l}=0.002$.

Results of signal modeling received from the distance $L=64$ wavelengths are presented in Figure 2. The first pulse corresponds to SP propagation at the dispersion absence $\left(\mathrm{T}_{r l}=0\right)$, and the second - to its presence. We well see that 
the SP shape is essentially distorted owing to the frequency dispersion of the medium parameters (see Figure $2 b$ ).

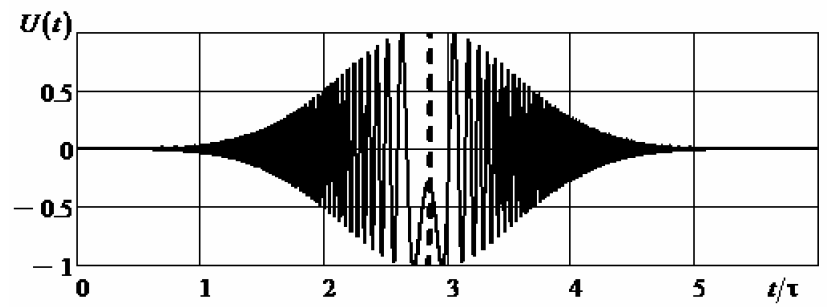

a)

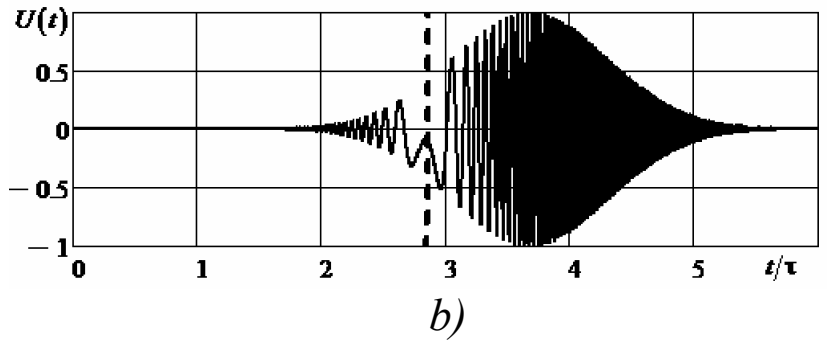

Figure 2. The signal in the receiver output at the absence (a) and at the presence (b) of the phase velocity dispersion, the dotted line corresponds to $t_{g r}=L / V_{g r}$

The delay of the first pulse is equal to $t_{g r}=L / V_{g r}=2.846$. The group delay time of the second pulse does not practically differ from $t_{g r}=2.846$. The error estimation in accordance with (2) gives $\delta_{\beta} \approx 3 \cdot 10^{-5}$. It would seem that the group velocity approximation may be fully used. However, the signal shape in Figure $2 b$ essentially differs from SP.

Distortions are the result of the frequency dependence of the damping index. For SP of the (10) type in the region $t>t_{g r}$, the instantaneous frequency is less than $f_{0}$ and the wave is less while for $t<t_{g r}$ the situation is opposite. The error estimation in accordance with (3) gives $\delta_{\alpha} \approx 0.5$. Obviously that the group velocity approximation cannot be used for the used SP and the medium parameters.

Thus, in spite of the fact that the group velocity is known, the distance determination to the object in the time domain is impossible.

Figure 3 shows the result of the numerical modeling of the above-described method of the object detection in the medium with the frequiency dispersion. The object is situated on the depth of 64 wavelength.

The plot of the mutual energy displaces directly in coordinates of the geometrical space. We see that the signal can be well compressed, which guarantees an accuracy of object position determination.

Modeling results of the stepwise operation mode for GPR are presented in Figures 4 and 5.
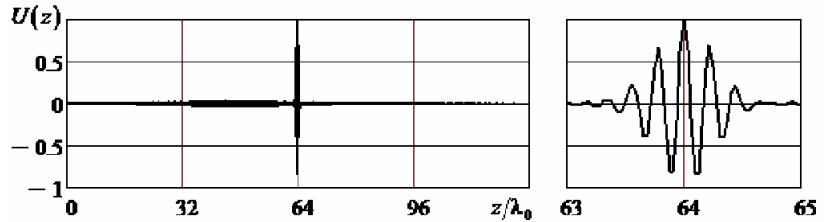

Figure 3. The modeling result of the method of distance determination to the object in the medium with the frequency dispersion

Figure 4 shows the totality of GPR routes at its displacements in the horizontal direction. The depth of the object bedding (the equivalent reflection point) is $z_{o b j}=24 \lambda_{0}$. The peculiarity of Figure 4 consists in the fact that the algorithm described presents routes directly in co-ordinates depth-displacement.

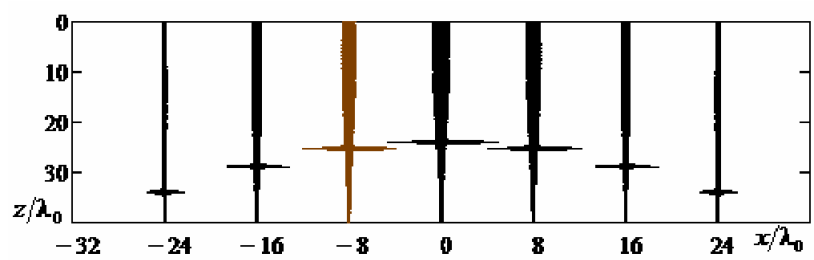

Figure 4. The set of GPR routes at its displacements along the horizontal axis

The result of the model signal processing in the form of the tonal image is presented in Figure 5.

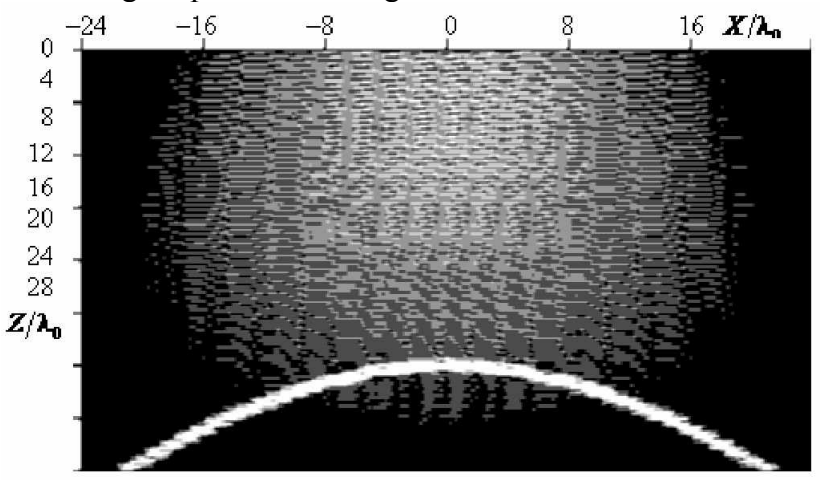

Figure 5. The graphical representation of modeling results.

As in Figure 4, the result presents directly in the geometrical space. The parabolic profile is clearly seen in the figure. Its peak corresponds to the object bedding depth.

\section{CONCLUSIONS}

We have shown a possibility to use the wideband chirp pulse signals in systems of ground penetrating radar sensing under conditions when we cannot apply the conception of the group delay time of the sensing pulses.

The offered method of signal processing allows the essential reduction of the influence of the frequency dispersion of medium properties upon an accuracy of the object position determination. Since the one of the distinguished features is the presentation of signal processing results of the geo-radar directly in natural geometrical space instead of "time- 
The Processing Algorithm for Wideband Signals

Propagating in Media with Frequency Dispersion horizontal displacement" space. The algorithm's peculiarity consists also in the fact that such data presentation is performed by the single pulse. Hence, using the set of such pulses, we can perform the data accumulation. This will permit to increase the geo-radar noise immunity and to improve its energy characteristics. In the aggregate, all this accelerates the analysis process of received data.

The method verification is performed by the numerical modeling, and the stepwise operation mode of the geo-radar is checked by the imitation on PC. Obtained results demonstrate a reliability of the object position determination.

This method can be spread to tasks related to the elastic wave propagation.

Thus, we can assume that the offered signal processing method of the wideband chirp pulse signals at the presence of medium dispersion will attract practical interest.

\section{REFERENCES}

[1] Günther Lehner. Electromagnetic Field Theory for Engineers and Physicists//Translated by Matt Horre, ISBN 978-3-540-76305-5, e-ISBN 978-3-540-76306-2, Springer Heidelberg, Dordrecht, London, New York. Springer-Verlag, Berlin, Heidelberg. 2010. DoI: $10.1007 / 978-3-540-76306-2$

[2] P. M. Kintner, B. M. Ledvina. The ionosphere, radio navigation, and global navigation satellite systems. Advances in Space Research, №35, 2005 .

[3] Ground Penetrating Radar Theory And Applications // Edited By Harry M. Jol. Elsevier Science, First Edition. 2009.

[4] Problems of ground penetrating radar technology (in Russian). Collective book / Under edition of A.Yu. Griniov. - Moscow: Radiotekhnika Publ., 2005. 416 p.: (Series "Radar Technology")

[5] A.P. Annan, Transmission Dispersion and GPR. JEEG, v.0, №2, January 1996, p. 125-136

[6] Parametric study on processing GPR signals to get a dispersion curve X. Xiao, A.Ihamouten, G. Villain, X. Dérobert. 15th International Conference on Ground Penetrating Radar. GPR 2014, June 30 - July 4, 2014 Square Brussels Meeting Centre, Brussels, Belgium.

[7] Detection and Target-Shape Recognition in Lossy and Dispersive Soil. Khalid M. Ibrahim1, Y, Khalid F. A. Hussein, and Abd-ElHadi A. Amman Land-Buried Object Progress In Electromagnetics Research B, Vol. 57, 279-298, 2014

[8] S. I. Baskakov, Signals and Circuits, Mir Publishers, Moscow, 1987.

[9] Mathematical Handbook For Scientists And Engineers Definitions, Theorems And Formulas. $2^{\text {nd }}$ edition. New York: McGraw-Hill, 1968.

[10] Application of Ground Penetrating Radar to Civil and Geotechnical Engineering. Richard J. Yelf, Electromagnetic Phenomena, V.7, №1 (18), 2007

[11] LFM ground penetrating radar with increased resolution capability (in Russian) A.S. Lopatchenko, I.Yu. Malevich, S.A. Savenko. Belorusia State University of Informatics and radio-electronics Publ. №3, 2015, 9. pp.43-48.

[12] Chun An Tsai, Rebecca Ghent, Alexander Boivin, and Dylan Hickson, "Discrimination of dispersive materials from radar signals using Q*," Ground Penetrating Radar, Volume 2, Issue 1, March 2019, pp. 26-50, Dor: 10.26376/GPR2019EP1/

[13] Frequency-dependent attenuation analysis of groundpenetrating radar data. John H. Bradford. Geophysics, vol. 72, №3 _may-june 2007; p. 7-16, 8 figs., 1 table. DoI: 10.1190/1.2710183

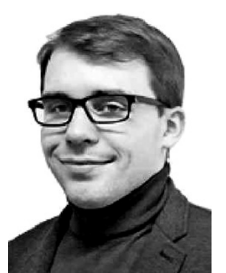

Andrei A. KALSHCHIKOV, born in 1994 in Ufacity (Russia). Graduated with honor in 2016 Nationa Research University "Moscow Power Engineering Institute" in specialty "Radio Electronic Systems and Complexes". From 2016 he is the PhDstudent of the Radio Engineering Fundamentals Department in NRU MPEI. He is engaged in development of compensation methods for signal distortions in transmission lines with the phase speed dispersion. He has several publications in Russian scientific journals. Worked in 2015 as a technician in R\&D Institute of Radio Engineering, and from 2016 fulfils the job of the engineer in the Department of A-D converters and signal processing. Participated in R\&D projects and testing of new radar stations. The current interests are fight with active noisy interferences, processing of moving targets, equalization of multi-channel systems, matched filtering, correction of signal propagating in media with the phase speed dispersion.

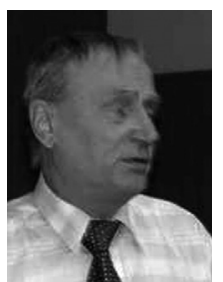

Vitaly V. SHTYKOV graduated from Radio Engineering Faculty of Moscow Power Engineering Institute (Technical University) in 1963. He had got Ph.D. (Techn.) in 1970. From 1963 he was a teacher of Department of Fundamentals of Radio Engineering of MPEI. Now he works as a full-professor of this department. He deliveres various lecture courses in Theoretical Fundamentals of Radio Engineering, Radio Engineering Circuits and Signals, Electrodynamics. He prepared lecture courses in Advanced Physics, Radio Physics, Functional Electronics, Acoustic-Electronics, Quantum Electronics, Biophysics. He has more than 200 scientific and academic publications, 21 patents. Scientific results of V.V. Shtykov are connected with investigations of new physical principles of signal detection and processing. He examined problems of quantum electronics, nonlinear phenomena in the plasma of gas discharge and semiconductors, in ferrimagnetic media, and also in the field of acoustic electronics.

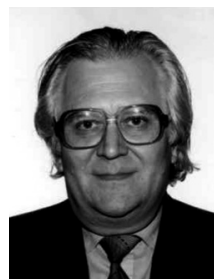

Sergey M. SMOLSKIY, born in 1946, Ph.D. in Engineering, Dr.Sc. in Engineering, full professor of Department of Radio Signals Formation and Processing of the National Research University "MPEI". He was engaged in theoretical and practical problems of development of transmitting cascades of short-range radar. In 1993 defended the Doctor of Science thesis and now he works as a professor of Radio Signals Formation and Processing Dept. Academic experience - over forty years. The list of scientific works and inventions contains over three hundreds of scientific papers, 15 books, more than 100 technological reports on various conferences, including international The active member of International Academy of Informatization, International Academy of Electrotechnical Sciences, International Academy of Sciences of Higher Educational Institutions. The active member of IEEE. The scientific work for the latter fifteen years is connected with conversion directions of short-range radar systems, radio measuring systems for fuel and energy complex, radio monitoring system etc. 


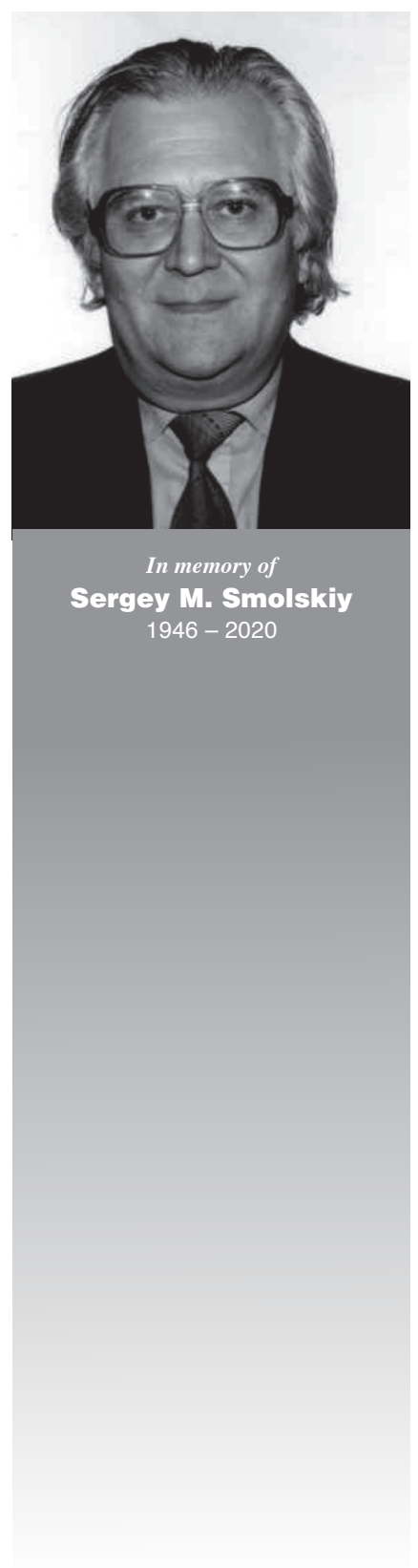

\section{Sergey M. Smolskiy $1946-2020$}

Sergey M. Smolskiy passed away on April 29 2020. He was a great professional with a rich creative, scientific, pedagogical and organizational biography, Honorary Academician of the Academy of Electrotechnical Sciences of the Russian Federation, Honorary Worker of Professional Education of the Russian Federation, Eminent Radio Engineer of the Russian Federation, Doctor of Technical Sciences, Emeritus Professor of the National Research University "Moscow Power Engineering Institute".

Sergey M. Smolskiy was born on January 2, 1946 in Moscow. In 1964 he entered the faculty of Radio Engineering of Moscow Power Engineering Institute which he graduated from in 1970 with a degree in Radio Physics. Already in his student years he became an $R \& D$ staff member of the department of radio transmitting devices and showed a propensity for scientific research and development. Under the guidance of V.M. Bogachev he achieved his first significant results. In 1970-1973 he pursued his postgraduate studies at MPEI with excellent academic performance.

After graduating and obtaining the degree of Candidate of Technical Sciences in 1974, he continued scientific work in the same department on theoretical and practical issues of radio transmitters, problems of the theory of frequency-modulated generators and autodyne. He was a responsible executor and scientific adviser of numerous research projects, including those carried out according to the decrees of the Government of the USSR.

Particular mention should be made of the last almost twenty-year period of Sergey $M$. Smolskiy's life. It was a period of his serious illness and at the same time a period of amazing creative activity.

Professor of the Department of Signal Formation and Processing since 2006 S.M. Smolskiy worked continuously until the last days of his life. He was the scientific director of a number of competitive projects of the Russian Foundation for Basic Research, Russian Scientific Foundation and the Ministry of Education and Science of the Russian Federation, he was a member of the Organizing Committee of a number of annual international scientific conferences and constantly interacted with colleagues from MPEI and other domestic and foreign universities and scientific organizations. During this period the number of scientific articles published by him doubled (more than 300), 15 original voluminous books were published in co-authorship, 11 of them were published in English in leading foreign scientific publishing houses. The latest book on quantum electronics and nonlinear optics, co-authored with Professor V.V. Shtikov, was released in early 2020.

In addition, Sergey M. Smolskiy translated from English into Russian and published 3 original books by foreign authors with a total volume of more than 1000 pages. At least 6 books translated by him into Russian with a total volume of about 2000 pages have not yet found their publishers, but are in use by colleagues.

The loss of Sergey M. Smolskiy is irreparable. The memory of a talented scientist, teacher, organizer and simply amazing, friendly, caring person will forever remain in our hearts. 